Jin-Ke Jiang, Gang Yang, Li-Ying Sun, Chun-Yan Wang and Feng Wang*

\title{
Crystal structure of poly[(5-bimethylamino-1-naphthalenesulfonato- $\mathrm{K} O$ )- $\left(\mu_{3}\right.$-hexamethylenetetramino- $\left.\mathrm{K}^{3} N: N^{\prime}: N^{\prime \prime}\right)$ silver(I)] dihydrate, $\mathrm{C}_{36} \mathrm{H}_{52} \mathrm{Ag}_{2} \mathrm{~N}_{10} \mathrm{O}_{8} \mathrm{~S}_{2}$
}

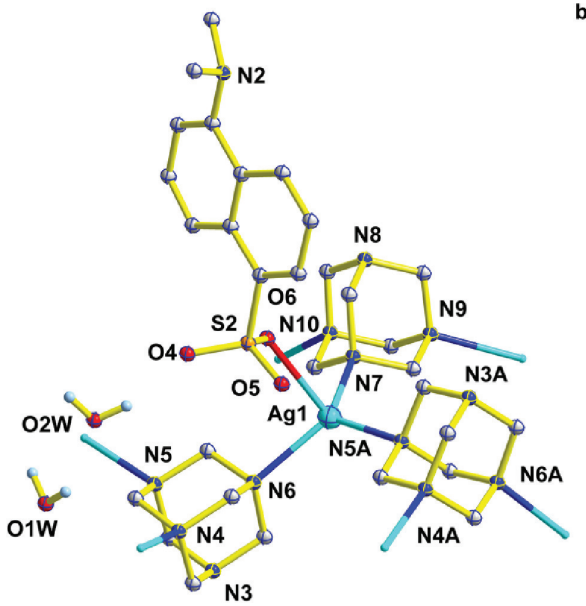

https://doi.org/10.1515/ncrs-2020-0109

Received February 24, 2020; accepted March 27, 2020; available online April 10, 2020

\section{Abstract \\ $\mathrm{C}_{36} \mathrm{H}_{52} \mathrm{Ag}_{2} \mathrm{~N}_{10} \mathrm{O}_{8} \mathrm{~S}_{2}$, orthorhombic, Pna2 $2_{1} \quad$ (no. 33), $a=11.7911(16) \AA, \quad b=31.310(5) \AA, \quad c=10.6883(11) \AA$, $V=3945.9(9) \AA^{3}, Z=4, R_{\mathrm{gt}}(F)=0.0353, w R_{\text {ref }}\left(F^{2}\right)=0.0784$, $T=293(2) \mathrm{K}$.}

\section{CCDC no.: 1985662}

Table 1 contains crystallographic data and Table 2 contains the list of the atoms including atomic coordinates and displacement parameters.

\section{Experimental details}

Data of a single crystal were collected via CrysAlis ${ }^{\mathrm{PRO}}$ at $293 \mathrm{~K}$ [1], structure was solved by SHELXT [2] within WinGX [3], refined by SHELXL [4] and the graphics were finished using Diamond [5].

*Corresponding author: Feng Wang, College of Biology and Pharmaceutical Engineering, Jilin Agricultural Science and Technology University, Jilin 132101, Province Jilin, P.R. China, e-mail: jlnkwf@126.com. https://orcid.org/0000-0001-8947-9297 Jin-Ke Jiang, Gang Yang, Li-Ying Sun and Chun-Yan Wang: College of Biology and Pharmaceutical Engineering, Jilin Agricultural Science and Technology University, Jilin 132101, Province Jilin, P.R. China

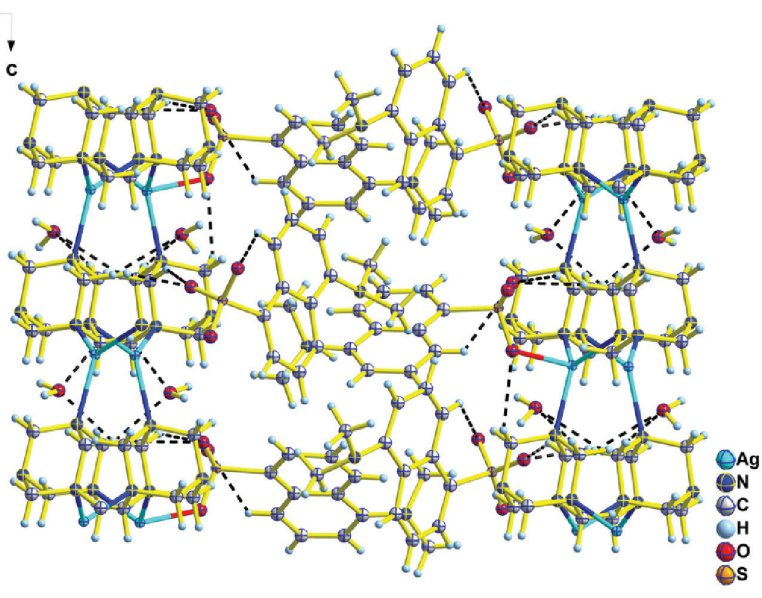

Table 1: Data collection and handling.

\begin{tabular}{|c|c|}
\hline Crystal: & Colourless block \\
\hline Size: & $0.24 \times 0.20 \times 0.17 \mathrm{~mm}$ \\
\hline Wavelength: & Mo $K \alpha$ radiation $(0.71073 \AA$ A $)$ \\
\hline$\mu:$ & $1.16 \mathrm{~mm}^{-1}$ \\
\hline Diffractometer, scan mode: & XtaLAB Pro, $\omega$ \\
\hline$\theta_{\max }$, completeness: & $25.0^{\circ}, 99 \%$ \\
\hline$N(h k l)_{\text {measured }}, N(h k l)_{\text {unique }}, R_{\text {int }}:$ & $18516,6557,0.039$ \\
\hline Criterion for $I_{\mathrm{obs}}, N(h k l)_{\mathrm{gt}}$ : & $I_{\text {obs }}>2 \sigma\left(I_{\text {obs }}\right), 5756$ \\
\hline$N(\text { param })_{\text {refined }}:$ & 533 \\
\hline Programs: & $\begin{array}{l}\text { CrysAlis }^{\text {PRO }}[1] \text {, SHELX [2, 4], } \\
\text { WinGX/ORTEP [3], Diamond [5] }\end{array}$ \\
\hline
\end{tabular}

Table 2: Fractional atomic coordinates and isotropic or equivalent isotropic displacement parameters $\left(\AA^{2}\right)$.

\begin{tabular}{lrrrr}
\hline Atom & $\boldsymbol{x}$ & $\boldsymbol{y}$ & $\boldsymbol{z}$ & $\boldsymbol{U}_{\text {iso }} / \boldsymbol{U}_{\text {eq }}$ \\
\hline C1 & $0.8936(7)$ & $0.4023(2)$ & $0.0835(8)$ & $0.0415(19)$ \\
C2 & $0.8990(6)$ & $0.4128(2)$ & $0.2067(11)$ & $0.0465(17)$ \\
H38 & 0.845740 & 0.401785 & 0.262110 & $0.056^{*}$ \\
C3 & $0.9831(9)$ & $0.4399(3)$ & $0.2508(8)$ & $0.058(2)$ \\
H37 & 0.986886 & 0.445976 & 0.335882 & $0.070^{*}$ \\
C4 & $1.0592(8)$ & $0.4575(3)$ & $0.1725(7)$ & $0.050(2)$ \\
H39 & 1.116804 & 0.474631 & 0.204336 & $0.060^{*}$ \\
C5 & $1.0526(7)$ & $0.4502(2)$ & $0.0426(7)$ & $0.0393(17)$ \\
C6 & $0.9702(7)$ & $0.4210(2)$ & $-0.0026(7)$ & $0.0354(17)$ \\
C7 & $0.9696(7)$ & $0.4119(3)$ & $-0.1325(8)$ & $0.0443(19)$ \\
H42 & 0.913867 & 0.394310 & -0.166044 & $0.053^{*}$ \\
C8 & $1.0498(8)$ & $0.4289(3)$ & $-0.2076(8)$ & $0.051(2)$ \\
H41 & 1.051221 & 0.421078 & -0.291539 & $0.062^{*}$
\end{tabular}


Table 2 (continued)

\begin{tabular}{|c|c|c|c|c|}
\hline Atom & $x$ & $y$ & $z$ & $U_{\text {iso }} * / U_{\text {eq }}$ \\
\hline $\mathrm{C} 9$ & $1.1310(8)$ & $0.4577(3)$ & $-0.1633(8)$ & $0.047(2)$ \\
\hline $\mathrm{H} 40$ & 1.185798 & 0.468418 & -0.217418 & $0.056^{\star}$ \\
\hline C10 & $1.1301(7)$ & $0.4702(3)$ & $-0.0417(7)$ & $0.0421(18)$ \\
\hline C11 & $1.1556(9)$ & $0.5395(3)$ & $0.0584(10)$ & $0.063(3)$ \\
\hline H43 & 1.207404 & 0.551422 & 0.118141 & $0.094^{\star}$ \\
\hline H44 & 1.085629 & 0.532378 & 0.099338 & $0.094^{\star}$ \\
\hline $\mathrm{H} 45$ & 1.141293 & 0.559914 & -0.006658 & $0.094^{\star}$ \\
\hline C12 & $1.3028(8)$ & $0.5111(4)$ & $-0.0746(11)$ & $0.071(3)$ \\
\hline $\mathrm{H} 46$ & 1.354808 & 0.528794 & -0.028804 & $0.107^{*}$ \\
\hline $\mathrm{H} 47$ & 1.277862 & 0.526045 & -0.148145 & $0.107^{\star}$ \\
\hline $\mathrm{H} 48$ & 1.340031 & 0.485131 & -0.098709 & $0.107^{\star}$ \\
\hline C13 & $0.3356(6)$ & $0.4125(2)$ & $0.5543(7)$ & $0.0355(16)$ \\
\hline C14 & $0.2435(7)$ & $0.4306(3)$ & $0.4986(7)$ & $0.045(2)$ \\
\hline $\mathrm{H} 25$ & 0.190922 & 0.413560 & 0.457188 & $0.054^{\star}$ \\
\hline C15 & $0.2279(7)$ & $0.4749(3)$ & $0.5036(8)$ & $0.046(2)$ \\
\hline $\mathrm{H} 26$ & 0.164019 & 0.487371 & 0.467816 & $0.055^{\star}$ \\
\hline C16 & $0.3071(7)$ & $0.4996(3)$ & $0.5614(8)$ & $0.043(2)$ \\
\hline $\mathrm{H} 27$ & 0.295899 & 0.528957 & 0.565273 & $0.052^{\star}$ \\
\hline C17 & $0.4054(6)$ & $0.4821(2)$ & $0.6155(7)$ & $0.0354(17)$ \\
\hline C18 & $0.4193(7)$ & $0.4372(2)$ & $0.6165(7)$ & $0.0362(17)$ \\
\hline C19 & $0.5138(7)$ & $0.4203(3)$ & $0.6798(7)$ & $0.045(2)$ \\
\hline $\mathrm{H} 28$ & 0.526294 & 0.390931 & 0.678218 & $0.054^{\star}$ \\
\hline $\mathrm{C} 20$ & $0.5867(7)$ & $0.4456(3)$ & $0.7427(8)$ & $0.053(2)$ \\
\hline $\mathrm{H} 29$ & 0.645907 & 0.433435 & 0.787839 & $0.064^{\star}$ \\
\hline $\mathrm{C} 21$ & $0.5739(8)$ & $0.4897(3)$ & $0.7407(7)$ & $0.052(2)$ \\
\hline $\mathrm{H} 30$ & 0.625553 & 0.506725 & 0.783460 & $0.062^{\star}$ \\
\hline $\mathrm{C} 22$ & $0.4878(7)$ & $0.5082(3)$ & $0.6776(7)$ & $0.0417(19)$ \\
\hline $\mathrm{C} 23$ & $0.5012(9)$ & $0.5709(3)$ & $0.5515(9)$ & $0.062(3)$ \\
\hline $\mathrm{H} 31$ & 0.465039 & 0.554803 & 0.486237 & $0.093^{*}$ \\
\hline $\mathrm{H} 32$ & 0.581974 & 0.569934 & 0.540153 & $0.093^{*}$ \\
\hline H33 & 0.475911 & 0.599999 & 0.548201 & $0.093^{*}$ \\
\hline $\mathrm{C} 24$ & $0.5280(12)$ & $0.5769(4)$ & $0.7693(9)$ & $0.080(4)$ \\
\hline H34 & 0.504867 & 0.566309 & 0.849631 & $0.120^{\star}$ \\
\hline H35 & 0.507474 & 0.606444 & 0.761987 & $0.120^{*}$ \\
\hline H36 & 0.608703 & 0.573987 & 0.760832 & $0.120^{*}$ \\
\hline $\mathrm{C} 25$ & $0.4003(8)$ & $0.1671(3)$ & $0.4466(8)$ & $0.0372(19)$ \\
\hline $\mathrm{H} 3$ & 0.327391 & 0.161023 & 0.408570 & $0.045^{\star}$ \\
\hline $\mathrm{H} 4$ & 0.456171 & 0.148599 & 0.407727 & $0.045^{\star}$ \\
\hline $\mathrm{C} 26$ & $0.5401(6)$ & $0.2209(2)$ & $0.4833(5)$ & $0.0262(14)$ \\
\hline $\mathrm{H} 7$ & 0.559923 & 0.250701 & 0.470690 & 0.031 * \\
\hline $\mathrm{H} 8$ & 0.598628 & 0.203617 & 0.444756 & $0.031^{*}$ \\
\hline $\mathrm{C} 27$ & $0.3429(6)$ & $0.2386(2)$ & $0.4824(6)$ & $0.0278(15)$ \\
\hline $\mathrm{H} 11$ & 0.270158 & 0.232898 & 0.443339 & $0.033^{*}$ \\
\hline $\mathrm{H} 12$ & 0.360556 & 0.268518 & 0.469921 & $0.033^{\star}$ \\
\hline $\mathrm{C} 28$ & $0.5038(6)$ & $0.1668(2)$ & $0.6351(7)$ & $0.0350(17)$ \\
\hline $\mathrm{H} 5$ & 0.561294 & 0.148535 & 0.598192 & $0.042^{\star}$ \\
\hline $\mathrm{H} 6$ & 0.499867 & 0.160291 & 0.723728 & $0.042^{\star}$ \\
\hline C29 & $0.3078(6)$ & $0.1841(2)$ & $0.6337(7)$ & $0.0380(17)$ \\
\hline $\mathrm{H} 1$ & 0.301868 & 0.177479 & 0.722126 & $0.046^{\star}$ \\
\hline $\mathrm{H} 2$ & 0.235133 & 0.177915 & 0.595032 & $0.046^{\star}$ \\
\hline C30 & $0.4473(5)$ & $0.2383(2)$ & $0.6726(6)$ & $0.0260(15)$ \\
\hline $\mathrm{H} 9$ & 0.466120 & 0.268136 & 0.660039 & 0.031 * \\
\hline $\mathrm{H} 10$ & 0.444066 & 0.233154 & 0.762039 & $0.031^{*}$ \\
\hline C31 & $0.2515(8)$ & $0.3406(2)$ & $0.9520(8)$ & $0.0325(17)$ \\
\hline $\mathrm{H} 13$ & 0.322858 & 0.348404 & 0.913132 & $0.039 *$ \\
\hline H14 & 0.192332 & 0.358291 & 0.916141 & $0.039^{*}$ \\
\hline $\mathrm{C} 32$ & $0.3193(6)$ & $0.2697(2)$ & $0.9818(6)$ & $0.0263(14)$ \\
\hline
\end{tabular}

Table 2 (continued)

\begin{tabular}{|c|c|c|c|c|}
\hline Atom & $x$ & $y$ & $z$ & $U_{\text {iso }}{ }^{*} / U_{\text {eq }}$ \\
\hline H17 & 0.306279 & 0.239662 & 0.965005 & $0.032^{*}$ \\
\hline H18 & 0.390800 & 0.277663 & 0.943303 & $0.032^{\star}$ \\
\hline C33 & $0.2151(5)$ & $0.2654(2)$ & $1.1742(6)$ & $0.0280(15)$ \\
\hline H23 & 0.199399 & 0.235403 & 1.159200 & $0.034^{*}$ \\
\hline H24 & 0.218624 & 0.269715 & 1.263977 & $0.034^{*}$ \\
\hline C34 & $0.1511(6)$ & $0.3364(2)$ & $1.1435(6)$ & $0.0310(15)$ \\
\hline $\mathrm{H} 21$ & 0.091452 & 0.354331 & 1.109545 & $0.037^{\star}$ \\
\hline H22 & 0.155158 & 0.341669 & 1.232861 & $0.037^{\star}$ \\
\hline C35 & $0.3479(6)$ & $0.3222(2)$ & $1.1403(6)$ & $0.0317(16)$ \\
\hline H15 & 0.352379 & 0.327438 & 1.229589 & $0.038^{*}$ \\
\hline H16 & 0.419943 & 0.330136 & 1.103266 & $0.038^{*}$ \\
\hline C36 & $0.1182(6)$ & $0.2841(2)$ & $0.9859(6)$ & $0.0262(14)$ \\
\hline H19 & 0.057998 & 0.301234 & 0.949773 & $0.031^{*}$ \\
\hline $\mathrm{H} 2 \mathrm{O}$ & 0.100949 & 0.254293 & 0.969724 & 0.031 * \\
\hline $\mathrm{Ag} 1$ & $0.21235(4)$ & $0.27894(2)$ & $0.71427(6)$ & $0.03515(13)$ \\
\hline Ag2 & $-0.04739(4)$ & $0.27043(2)$ & $1.21066(6)$ & $0.05288(19)$ \\
\hline N1 & $1.2047(6)$ & $0.5011(2)$ & $0.0043(7)$ & $0.0514(18)$ \\
\hline N2 & $0.4723(7)$ & $0.5526(2)$ & $0.6719(6)$ & $0.0508(19)$ \\
\hline N3 & $0.3945(5)$ & $0.1575(2)$ & $0.5775(6)$ & $0.0376(15)$ \\
\hline N4 & $0.4309(5)$ & $0.21218(19)$ & $0.4216(5)$ & $0.0279(12)$ \\
\hline N5 & $0.5370(5)$ & $0.21191(18)$ & $0.6186(5)$ & $0.0249(12)$ \\
\hline N6 & $0.3346(5)$ & $0.22975(18)$ & $0.6179(5)$ & $0.0272(12)$ \\
\hline N7 & $0.2267(5)$ & $0.29519(18)$ & $0.9258(5)$ & $0.0257(12)$ \\
\hline N8 & $0.2581(5)$ & $0.34818(18)$ & $1.0866(5)$ & $0.0329(14)$ \\
\hline N9 & $0.1220(5)$ & $0.29132(18)$ & $1.1221(5)$ & $0.0271(12)$ \\
\hline N10 & $0.3266(4)$ & $0.27648(18)$ & $1.1181(5)$ & $0.0241(12)$ \\
\hline 01 & $0.8779(6)$ & $0.3269(2)$ & $0.0005(11)$ & $0.092(3)$ \\
\hline 02 & $0.7288(5)$ & $0.3744(2)$ & $-0.0579(7)$ & $0.0673(18)$ \\
\hline $01 \mathrm{~W}$ & $0.5611(8)$ & $0.3159(2)$ & $-0.1566(9)$ & $0.085(2)$ \\
\hline $\mathrm{H} 1 \mathrm{~A}$ & 0.514823 & 0.334154 & -0.186478 & $0.128 *$ \\
\hline $\mathrm{H} 1 \mathrm{~B}$ & 0.604771 & 0.330523 & -0.109906 & $0.128 *$ \\
\hline 03 & $0.7408(7)$ & $0.3478(3)$ & $0.1495(7)$ & $0.091(3)$ \\
\hline $02 \mathrm{~W}$ & $0.5953(7)$ & $0.3075(2)$ & $0.3213(8)$ & $0.074(2)$ \\
\hline $\mathrm{H} 2 \mathrm{~A}$ & 0.644400 & 0.326127 & 0.300748 & $0.111^{*}$ \\
\hline $\mathrm{H} 2 \mathrm{~B}$ & 0.535562 & 0.321851 & 0.336104 & $0.111^{*}$ \\
\hline 04 & $0.4576(6)$ & $0.34722(19)$ & $0.4915(6)$ & $0.0614(18)$ \\
\hline 05 & $0.2535(7)$ & $0.3422(2)$ & $0.4683(8)$ & $0.065(2)$ \\
\hline 06 & $0.3418(6)$ & $0.34098(18)$ & $0.6726(5)$ & $0.0630(19)$ \\
\hline S1 & $0.80208(17)$ & $0.35937(6)$ & $0.0399(2)$ & $0.0470(5)$ \\
\hline S2 & $0.34806(19)$ & $0.35643(6)$ & $0.5454(2)$ & $0.0445(5)$ \\
\hline
\end{tabular}

\section{Experimental detail}

Hydrogen atoms of the organic ligands are positioned using a riding model [4]. The coordinates of the hydrogen atoms of the water molecules are refined freely. $U_{\text {iso }}$ parameters of all hydrogen atoms are constrained depending of the attached parent atoms.

\section{Comment}

As is depicted in the left part of the figure, the Ag1 is four-coordinated by three nitrogen atoms and one oxygen atom. And three nitrogen atoms come from three hexamethylenetetramine ligands, one oxygen atom comes 
from 5-bimethylamino-1-naphthalenesulfonate. The bond distances of $\mathrm{Ag}-\mathrm{O}$ and $\mathrm{Ag}-\mathrm{N}$ are in the normal ranges. Additionally, there are two free water molecules. Most importantly, the weak interaction $\mathrm{CH}$. . O also plays an important role in forming a 3D structure [6-8] (cf. right part of the figure). (symmetry codes: $\mathrm{A}=-1 / 2+x, 1 / 2-y, z)$.

Acknowledgements: This work was supported by the Youth Foundation of Jilin Agricultural Science and Technology University (no. 201811439036).

\section{References}

1. Rigaku Corporation: CrysAlis ${ }^{\mathrm{PRO}}$. Rigaku Corporation, Yarnton, Oxfordshire, England (2015).

2. Sheldrick, G. M.: SHELXT - integrated space-group and crystalstructure determination. Acta Crystallogr. A71 (2015) 3-8.
3. Farrugia, L. J.: WinGX and ORTEP for Windows: an update. J. Appl. Crystallogr. 45 (2012) 849-854.

4. Sheldrick, G. M.: Crystal structure refinement with SHELXL. Acta Crystallogr. C71 (2015) 3-8.

5. Brandenburg, K.: DIAMOND. Visual Crystal Structure Information System. Ver. 3.2., Crystal Impact, Bonn, Germany (2012).

6. Wu, H.; Dong, X.-W.; Ma, J.-F.; Liu, H.-Y.; Yang, J.; Bai, H.-Y.: Influence of anionic sulfonate-containing and nitrogen-containing mixed-ligands on the structures of silver coordination polymers. Dalton Trans. 17 (2009) 3162-3174.

7. Jiang, J.-K.; Li, X.-X.; Jiang, H.; Dong, X.-W.: Crystal structure of poly[aqua $\left(\mu_{2}\right.$-2-amino-1,4-benzenedisulfonato- $\mathrm{K}^{3} \mathrm{~N}, \mathrm{O}$ : $\left.O^{\prime}\right)$ - $\left(\mu_{4}\right.$-hexamethylenetetramino- $\left.{ }^{4} N: N^{\prime}: N^{\prime \prime}: N^{\prime \prime \prime}\right)$ disilver(l)] monohydrate, $\mathrm{C}_{12} \mathrm{H}_{21} \mathrm{Ag}_{2} \mathrm{~N}_{5} \mathrm{O}_{8} \mathrm{~S}_{2}$. Z. Kristallogr. NCS 234 (2019) 1251-1252.

8. Liu, F.-J.; Sun, D.; Hao, H.-J.; Huang, R.-B.; Zheng, L.-S.: A novel photolumine scent silver(I) wire supported by 4tertbutylbenzoate and ligand-unsupported Ag-Ag interactions. Inorg. Chem. Commun. 15 (2012) 136-139. 